\title{
ESBOZO DE UN ESTUDIO DE ACTITUDES LINGÜÍSTICAS EN EL SALVADOR
}

\author{
José Roberto Alexander Quintanilla \\ Butler University
}

\section{RESUMEN}

Este estudio analiza las valoraciones de los salvadoreños hacia algunos fenómenos lingüísticos. Los medios oficiales y el sistema educativo en este país promueven el uso de un español acercado al habla de Castilla (conocida en este país como «español de España») por poseer rasgos lingüísticos prestigiosos, como la distinción fonológica entre $s$ y $z$ y el mantenimiento de $s$ a final de sílaba. Esto ha creado una dicotomía de sentimientos hacia el español local. 100 participantes brindaron su valoración sobre algunos fenómenos lingüísticos de ambas variedades. Los resultados muestran que los salvadoreños tienen actitudes positivas hacia el español salvadoreño, a pesar de considerar que el español de España es «mejor» y de evaluar negativamente algunos fenómenos tanto locales como peninsulares. Esto revela que en esta comunidad hay signos de inseguridad lingüística. Dicha inseguridad aumenta cuando entra en juego el nivel de educación de los hablantes

Palabras clave: actitudes, español salvadoreño, estigmatización, inseguridad lingüística.

\section{ABSTRACT}

This work analyzes the attitudes of Salvadorans towards some features of the Spanish of El Salvador and Spain. Salvadoran schools promote the Spanish of Castile (known by Salvadorans as «Spain's Spanish»). This can be the reason for the existence of two Spanish varieties (or the use of some of its elements) in some linguistic contexts of Salvadoran Spanish and the creation of myths and stigmatization towards some linguistic features of both varieties. 100 participants were interviewed in the city of San Salvador. Our results show that Salvadorans have positive attitudes to both Spanish varieties, but there still remains stigma towards some features of both Spanish varieties, which means there is linguistic insecurity among Salvadorans. Linguistic insecurity is higher when speakers have more education.

Key Words: Salvadoran Spanish, stigma, attitudes, peninsular Spanish, linguistic insecurity. 


\section{INTRODUCCIÓN}

Poco o nada se ha estudiado sobre el español salvadoreño de hoy en día. La escasa bibliografía de esta variedad de español nos llega desde antaño, de trabajos esmerados pero con poco rigor académico, dada la carencia de centros de investigación lingüística en este país. De estos trabajos, sobresale la aportación de Rivas 1978, quien dedicó un libro a las particularidades lingüísticas que, a su criterio, eran de origen salvadoreño. Rivas creía que muchos fenómenos lingüísticos propios de El Salvador procedían de un influjo náhuatl, incluso aquellos que también están presentes en España. Los desaciertos de Rivas no restan importancia a su esfuerzo, pues nos sirven para conocer algunos de los fenómenos lingüísticos que se podían reconocer en el habla salvadoreña en la época en que se recogieron estos datos. Dadas las circunstancias que señalamos al inicio, los trabajos que habían precedido y los que han sucedido a Rivas no han contado con el uso de métodos de investigación científicos y son más bien de tipo descriptivo. Quizá la primera descripción más fiable sobre el español salvadoreño sea la de Lipski 1994, sobre el español americano, donde hace una recopilación de los rasgos lingüísticos que caracterizan el habla de El Salvador.

Por otro lado, hay estudios que se han enfocado en el habla de los salvadoreños en el exterior, como el de Hernández 2002, que estudia la acomodación a otras variedades. Estos estudios no dejan de ser importantes para la dialectología salvadoreña, pero se alejan del contexto tanto geográfico como lingüístico que echamos de menos, es decir, el del español hablado en El Salvador. Carecemos de trabajos sobre el español salvadoreño que sean recientes, que nos muestren lo que está pasando a nivel lingüístico en esta variedad y que, además, se ajusten a metodologías más actualizadas y aplicables a contextos de habla reales (in situ).

El presente estudio ha sido motivado por esta desventurada falta y no pretende ser más que una mínima iniciación al estudio del español salvadoreño de nuestra época. Paradójicamente, dicha carencia de trabajos sobre El Salvador y su lengua nos da a la vez una infinidad de particularidades lingüísticas para estudiar en este territorio. Algunas de las particularidades del español salvadoreño son el uso del voseo (vos sos mi amigo), el uso de tú como forma de respeto en el habla escrita o cuando se habla con extranjeros, la presencia del artículo indefinido antes de un adjetivo posesivo (tengo una mi amiga) la aspiración de $s$ al final de sílaba (los amigos: /lohamigoh/), el jejeo (nosotros: /nohotros/), la presencia al final de sílaba del sonido fricativo palatal sordo sh (en palabras de origen autóctono o extranjero y con grafías ch o sh como carwash, mish, cash, Mitch, etc.), la $n$ velar al final de sílaba, la contracción de verdad como veá y va (¿veá que si??), etcétera. 
Como punto de partida, hemos decidido empezar indagando las actitudes de los salvadoreños hacia algunas de las particularidades de su propio español y del español que se les enseña como «correcto»en la escuela (en teoría el español de España). Esperamos que este esbozo se convierta en una invitación a otros interesados a explorar el español centroamericano y que, desde luego, nos permita conocer un poco del habla salvadoreña.

\section{MARCO TEÓRICO}

El estudio de las actitudes o creencias de los hablantes hacia la lengua es de vital importancia dentro de la sociolingüística. Además, las actitudes de los hablantes hacia un determinado rasgo lingüístico pueden ser una señal de un cambio lingüístico, como señala López 2001, p. 147.

Comenzamos este acercamiento con una evaluación de las actitudes de los salvadoreños hacia su propia variedad de español. Dada la carencia de estudios de tipo lingüístico realizados en esta región geográfica, nos vemos en la situación de no poseer precedentes que nos orienten a un enfoque más puntual. Hemos hecho, entonces, una selección de particularidades lingüísticas de este español y las hemos expuesto a un grupo de salvadoreños para saber sus reacciones, positivas o negativas, sobre estos fenómenos. Hablaremos con más detalle sobre la metodología en $\$ 3$. Hemos añadido igualmente fenómenos lingüísticos popularmente asociados en El Salvador con el «español de España» (cabe aclarar que en la práctica estos fenómenos se asemejan más a los de cualquier variedad del español del centro y norte de España, posiblemente la de Castilla).

No es raro escuchar a un salvadoreño decir que su español «no es español de verdad». La apreciación negativa hacia la lengua propia no es una casualidad, ya que en este país centroamericano el sistema educativo ha promovido una variedad de español extranjera, en teoría «el español de España». Aclaramos que lo que se conoce como el español de España en El Salvador no es más que una idealización de un español «correcto», pues no queda claro a qué variedad de español peninsular se inclinan más las autoridades educativas salvadoreñas. Asumimos que se refieren al español del centro y norte de España, el que conserva la distinción entre z y $s$ y preserva el pronombre vosotros. Podemos comprobar este supuesto con el hecho de que en las clases de lenguaje de la escuela primaria y secundaria de este país se enseña a los alumnos a conjugar los verbos con el pronombre vosotros, a pesar de que este es totalmente inexistente no solo en El Salvador sino también en todo el continente americano. Queda claro que la idealización que se tiene en El Salvador sobre el «español de España» no es más que un estereotipo, 
basado en una serie de elementos lingüísticos que coinciden con las variedades habladas en el centro y norte de España.

La idolatría hacia rasgos lingüísticos del español de España y las impresiones que se suelen oír de los mismos salvadoreños hacia su propia lengua podrían ser un indicio de que poseen algún complejo de inseguridad o inferioridad lingüística. Lipski 1986, p. 104, llama a esta dicotomía, la que surge entre la posición oficial (a favor del español de España) y el día a día (la ausencia de rasgos españoles en el habla espontánea de los salvadoreños) «esquizoglosia», un término original de Haugen 1962. La esquizoglosia se observa cuando los hablantes titubean en el uso de elementos lingüísticos locales y los de otra variedad, a la que ellos consideran «superior» o «más correcta». Dicha vacilación ya ha sido notada por Quintanilla 2009 en los hablantes salvadoreños, cuando adoptan el tuteo al hablar con extranjeros, es decir, el uso de tú en vez de vos como pronombre de segunda persona singular, o cuando intentan acomodarse lingüísticamente al español mexicano (en situaciones de contacto) y evitan, por ejemplo, aspirar la $s$, esto último notado por Hernández 2002, entre salvadoreños en Estados Unidos.

Observamos, sin embargo, que a pesar de la intensa y longeva promoción del «español de España» en los textos escolares salvadoreños, el uso de estructuras o rasgos lingüísticos típicos y exclusivos de España son casi inexistentes en el habla cotidiana de este país. Volvemos a aclarar que cuando hablamos de español peninsular o de España nos referimos a una idealización de los salvadoreños de esta variedad, ya que, como sabemos, el español en España no es uniforme en absoluto. Como dijimos antes, los salvadoreños asocian el «español de España»con el uso del fonema interdental $/ \theta /$ (aunque muchos desconocen que el fonema es exclusivamente para la grafía $z$ y las combinaciones $c e$ y $c i$ y no para $s$ ). También se asocia con España el mantenimiento de /s/ (no aspiración) o con el sonido de /s/ apical, a veces confundido con $s h$, existente en el habla salvadoreña. Al tratar de imitar el «acento de España», probablemente un salvadoreño pronunciaría la palabra Espa$\tilde{n} a$ como 'Eshpaña'. Otro rasgo que los salvadoreños suelen relacionar con el habla de España es el uso del pronombre de segunda persona plural vosotros.

El español culto salvadoreño evita, en lo posible, utilizar rasgos lingüísticos que están estigmatizados en el español salvadoreño, como la aspiración de $s$, el jejeo y, en cierta medida, aunque con otras características, el voseo. En el habla espontánea, este tipo de habla rebuscada, que podría sonar también afectada, tiene poco éxito, especialmente en lo que respecta al voseo y la aspiración de $s$, pues prácticamente son rasgos omnipresentes en el habla de casi todos los salvadoreños. Las cosas cambian cuando los hablantes tienen más control de lo que van a decir o, en su defecto, suficientes conocimientos metalingüísticos. Nos referi- 
mos aquí a la publicidad, los programas de televisión (especialmente si están pregrabados), las actuaciones en público, la lengua escrita, discursos, etc. En estos contextos es posible encontrar el uso del tuteo y el mantenimiento de $s$ y a veces del pronombre vosotros, aunque este último más específicamente para citar textos religiosos o en juramentaciones o documentos legales (como suele suceder con el futuro del subjuntivo, casi extinto en el español moderno). Asimismo, no es raro oír a algunos maestros salvadoreños decir a sus alumnos que los pares de letras $b$ y $v, z$ y $s$, y el dígrafo $l l$ y la $y$ deben ser diferenciados en su pronunciación si se quiere hablar «español correcto».

Notamos que la concepción de español de España está, pues, muy idealizada y que el uso de rasgos de esa variedad es muy arbitrario y cambia según el registro, y se hace más énfasis en algunos usos más que en otros, a veces pasándose por alto algunos usos que deberían imitarse más, por ser más típicos del «español de España». Así vemos que en el español culto de El Salvador jamás se suele diferenciar la pronunciación de $s$ y $z$, aunque siga viéndose como una forma «correcta» de pronunciar.

Al parecer, la idolatría hacia el español de una región lejana no siempre se da en todas las regiones del mundo hispano, aunque haya cierta inseguridad lingüística dentro de una variedad local. En España, según nuestro conocimiento, no se concibe ninguna variedad americana como la «mejor», ni se trata de imitar ninguna variedad que no sea una de España, especialmente la del centro y norte. De acuerdo con HernándezCampoy 2003, p. 34, y Hernández-Campoy y Jiménez 2003, p. 342, el español septentrional parece ir ganando terreno en el sur de España. Una señal de que el español del norte y centro de España goza de cierto prestigio es el hecho de que los medios de comunicación (radio, TV, cine, literatura, doblaje de películas, etc.) que se ven nacionalmente en ese país tienden a hacerlo en esta variedad de español (y no, por ejemplo, en andaluz o en canario). En El Salvador, la influencia directa del español de España (cualquiera que sea la variedad) es muy poca. Un repaso por la programación de los canales de televisión salvadoreños nos muestra que las series y programas transmitidos son importados mayormente de países hispanoamericanos, especialmente de México, Colombia y Argentina (salvo la televisión por cable, donde suele incluirse un par de canales españoles entre más de un centenar de cadenas de Estados Unidos e Hispanoamérica). Se podría decir que el español de España solo llega a través de la música, donde tal vez pase desapercibido para un salvadoreño corriente. Esto es diferente en España, donde regiones como Andalucía o las Islas Canarias reciben mucha influencia lingüística de la región central a través de los medios de comunicación.

Fuera de los libros de texto en que se aprende a usar tú y vosotros, la idea de lo que es el español «correcto» de España es solo una abstracción. Como dijimos antes, la variedad de español que se oye con mucha 
frecuencia en los medios de comunicación salvadoreños es la mexicana. En El Salvador se transmite cada día una infinidad de telenovelas, programas de TV, películas, canciones, etc., de México. Casualmente, el español de México posee algunos rasgos en común con el español peninsular que para un salvadoreño pueden sonar como un español más «correcto». Por ejemplo, en México se usa el tuteo, que en El Salvador se aprende en la escuela, y la $s$ no se aspira (al menos en el español del centro de México), una pronunciación que está estigmatizada en el habla culta salvadoreña. No es raro, entonces, que algunos presentadores de televisión salvadoreños hablen con cierta influencia lingüística del español mexicano, tanto fonéticamente como a nivel léxico. La imitación del acento mexicano es tal, que a veces resultaría difícil saber si los que están presentando las noticias son salvadoreños o mexicanos. Muchas palabras o expresiones mexicanas como rola 'canción' o qué ondas 'qué tal' ya se han convertido en parte del habla salvadoreña y no hay duda de que son palabras naturales de México.

La adopción de rasgos del español peninsular por parte de americanos no es una novedad ni una exclusividad de los salvadoreños. Sarmiento, en el siglo XIX, se mofaba del poco éxito que tenían aquellos que imitaban el acento castellano en Chile, como relata Velleman 1997. El rasgo imitado más típico entre los chilenos intelectuales era el de la distinción entre $s$ y $z$ (como se hace de forma natural en el centro y norte de España). Sarmiento también señalaba que se intentaba hacer distinción fonética entre las letras $b$ y $v$. Como es sabido, en español no es usual dicha distinción, ni siquiera en España (los ejemplos que Lope Blanch 1988, y Stevens 2000 han encontrado de este uso en algunas partes de México y entre los profesores hispanos que enseñan español en Estados Unidos no son un rasgo general).

La aparente idealización entre los salvadoreños de que el mejor español es el de España no es azarosa. En primer lugar, el español se originó en España y esto hace creer a muchos salvadoreños que su español es prestado y que los verdaderos dueños son los españoles. Como consecuencia, los hablantes no se sienten dueños del idioma que hablan y muestran complejos que resultan en vacilación o ultracorrección en el uso de su propia lengua. Lara 2001, subraya esta situación al lamentar que cuando se habla de lenguas americanas en América, se entiende «las que son aborígenes», p. 31, de este continente, a pesar de que el español tanto como el inglés, entre otros idiomas americanos, son hoy en día tan americanos como europeos, pues son hablados en ambos continentes. La sensación de impropiedad de la lengua española es tal en los salvadoreños que estos llaman al español salvadoreño «caliche», en oposición al término «español».

En segundo lugar, existe la creencia entre algunos académicos de que el uso de neologismos o regionalismos lingüísticos en las varieda- 
des del español americano puede desencadenar la creación de muchas lenguas, dado el aislamiento que hay entre algunas regiones. Andrés Bello 1970, autor de la Gramática de la Lengua Castellana en el siglo XIX, 1948, temía que el español se convirtiera en una variedad de «dialectos», como sucedió con el latín, y que por lo tanto, los pueblos hispanoamericanos estarían incomunicados y limitados en su progreso económico. Esto llevó a Bello a luchar por un español americano basado en los rasgos del español culto de España, especialmente el español de los grandes escritores. Estas ideas de Bello se han mantenido hasta nuestros días, y, por lo tanto, vuelven a favorecer el habla de España. No es raro leer en los periódicos salvadoreños reacciones de desconcierto hacia nuevos usos de la lengua española que se oyen en El Salvador.

Finalmente, otro punto que le da ventaja al español de España es que El Salvador es un país que ha tenido poco protagonismo e influencia cultural a nivel regional desde el nacimiento y desarrollo de las naciones hispanoamericanas. La mayor parte del arte, música, literatura, etc., viene de México, Argentina, Colombia y otras naciones hispanas. La sensación de que lo «mejor» viene del exterior es posible que haya reforzado los sentimientos de inferioridad lingüística en los hablantes salvadoreños.

No obstante, también hay señales de que las actitudes de los salvadoreños hacia su propia lengua pueden estar cambiando. Un vistazo a los medios de comunicación locales en la actualidad nos muestra claros signos de vacilación entre fenómenos lingüísticos típicamente salvadoreños y aquellos no locales. Se observa, especialmente en los programas de corte juvenil, una mayor retención de la aspiración de /s/, un uso elevado del voseo, el uso del verbo poder con el significado de 'saber', más utilización de léxico y jerga local en contextos menos informales (como el uso de cabal, púchica, etc.). Estos son fenómenos que, por lo general, los salvadoreños conocen y a los que no costaría relacionar como lenguaje nacional o «caliche». Se oyen igualmente otros rasgos que posiblemente desconozcan los propios salvadoreños pero que son característicos del habla de este país, como la velarización de /n/, la pronunciación oclusiva de /b/, /d/ y /g/ después de consonante, el uso de hasta para referirse al inicio de un evento, entre otros. Todo esto hace que cada vez el habla de los medios parezca más salvadoreña.

La existencia de cambios de registro en el español de El Salvador, cada uno con una función diferente, podría parecer un caso de lo que Ferguson 1959, llama «diglosia». Numerosos hechos, como la función, el prestigio, la herencia literaria y la gramática de un idioma pueden llevar a una sociedad a dividir dos variedades o dos idiomas y ponerle una finalidad específica a cada uno. En dicha división, habría una variedad alta y otra baja, la primera prestigiosa y la otra no (a pesar de que 
esta última es la más usada en el habla espontánea). Vemos así que en El Salvador existe una división entre el español local, es decir, lo que llamaríamos español salvadoreño o «caliche» (con sus particularidades fonéticas, sintácticas, léxicas, etc.) y el español idealizado como de España. El español salvadoreño no difiere drásticamente, al igual que las demás variedades del español, de cualquier otra variedad del español, es decir que no se llega al punto de la incomprensión entre un hablante de El Salvador y otro de México. En ese sentido, no estamos hablando de dos lenguas o variedades muy distintas y, por lo tanto, no creemos que estemos ante un caso típico de diglosia. Sin embargo, el concepto de diglosia nos ilustra con bastante cercanía la relación que hay en El Salvador entre el español salvadoreño o «caliche» (hablado espontáneamente) y el español que promueve el sistema educativo salvadoreño, basado en ciertos rasgos del español de España (aunque solamente sea una idealización). La relación diglósica entre dos variedades del español ya ha sido también notada por Guitart 1996. Este autor compara el caso del español andaluz y del español con rasgos del centro de España (el autor llama a este último "castellano») en Andalucía. Al igual que en El Salvador, en Andalucía la escuela promueve el español «castellano» (o del centro de España), lo que ha provocado cierto tipo de diglosia entre dos variedades del mismo idioma. En esta diglosia, de acuerdo con el mismo autor, la variedad baja es el andaluz. Guitart hace una división entre lectos conservadores (variedad alta) y radicales (variedad baja), siendo estos últimos aquellos que muestran mayor variación consonántica, como la aspiración de /s/, velarización de /n/, etc. Guitart no cree que la promoción del español «castellano» en la educación influya en la inseguridad lingüística de los andaluces, ya que estos hablantes también muestran vacilación entre el uso de elementos lingüísticos andaluces. En cuanto al caso salvadoreño, la vacilación entre fenómenos lingüísticos típicos de El Salvador y de España se da más, y quizá exclusivamente, entre hablantes con cierto nivel de estudios. Como mencionamos antes, los contextos en que se intentaría elidir la aspiración de /s/ o usar menos el voseo sería en un español muy formal y en registros muy específicos, como dentro de los medios de comunicación, cuando se dramatiza o, aunque no de manera evidente en la parte fonética, cuando se escribe una carta. Casi nunca se hablaría «a la española» en el habla espontánea, como en una conversación entre amigos. Como mencionamos antes, fuera de la escuela, la promoción del español castizo es casi nula y, por lo tanto, es lógico pensar que es precisamente la escuela la que probablemente influya en la inseguridad lingüística observada en la población salvadoreña.

El apego hacia rasgos lingüísticos locales se observa de forma más clara en regiones como Buenos Aires, donde un porteño titubea menos a la hora de elegir el pronombre de segunda persona vos cuando se di- 
rige a un extranjero, mientras que es bastante posible que un salvadoreño intente usar tú, a pesar de que en el habla espontánea salvadoreña no sea precisamente ese el pronombre que utilizaría. En algunos países hispanos, incluso, se cree que el «mejor español»se habla localmente, como es el caso de Colombia, según Lipski 1994, p. 227, donde el español de Bogotá tiene la reputación de ser «más puro».

Lipski 1994, p. 157, cree que aquellas variedades que muestran mayor concordancia entre la ortografía y la pronunciación reciben mejor valoración por parte de muchos hablantes. En este sentido, el español peninsular (específicamente el del centro y norte de España) al hacer distinción entre los fonemas /s/ y / $\theta /$ y no aspirar /s/ se acerca más a la escritura. Tal vez esa sea una de las razones por las que el español del centro y norte de España se vea como «mejor» en El Salvador. Este mito de la ortografía también ha ayudado a que se piense que en español debería hacerse una distinción en la pronunciación de las letras $b$ y $v$, idea muy promulgada por muchos profesores en el pasado y probablemente todavía vigente hoy en día. Vemos que fenómenos como la aspiración de /s / muestra variación dialectal en todo el continente, especialmente entre mantenimiento, aspiración y elisión. Dado que la aspiración y la elisión no corresponden con el ideal de correspondencia entre la pronunciación y la ortografía, estos se han convertido en rasgos generalmente estigmatizados. Un ejemplo sería el español de Colombia, donde el habla de la zona central, con mantenimiento de /s/, tiene más prestigio que el de la zona costera, donde hay aspiración de /s/. Aún así, la idea de que la correspondencia con la ortografía es primordial para que un dialecto sea visto como mejor español no es general, pues el uso del fonema $/ \theta /$ no parece ser un rasgo que todos los hispanoamericanos quieran imitar.

Esta dicotomía entre actitudes positivas y negativas podría estar generando cambios lingüísticos interesantes en todo el mundo hispano. Antes se hablaba de un posible nacimiento de diversos dialectos como consecuencia de las diferencias lingüísticas que irían surgiendo en cada rincón del mundo hispano. Para ello había que promover un español castizo, tal y como hizo Andrés Bello. Hoy en día, sin embargo, autores como López 2000, creen que el temor a una división del español en varias lenguas ya ha desaparecido y que solo pertenece a tiempos pasados. Asimismo, Lipski 1986, mantiene que en América Central las actitudes populares hacia el voseo, un rasgo importante del español salvadoreño, comienzan a cambiar positivamente, gracias a los escritores y líderes populares que tienen sentimientos nacionalistas. Lipski 1994, p. 158, también piensa que el castellano «mitológico» está desapareciendo de los hábitos educativos. La Real Academia Española y las Academias de la lengua en América trabajan en conjunto en proyectos que unen el español a través de sus propias diferencias, como se observa con la 
creación de la importante obra Diccionario de americanismos en 2010. Si las actitudes oficiales parecen estar cambiando, nuestra pregunta es, ¿qué está pasando entre los hablantes, especialmente en el caso de El Salvador, de cuyo español sabemos poco?

Pensamos que nuestro trabajo puede brindar algunas primicias sobre lo que piensan los salvadoreños de su lengua, para lo cual nos hemos acercado a ellos y les hemos pedido su valoración sobre algunos de los fenómenos lingüísticos de su propia lengua y de lo que se conoce como el «español de España». Queremos saber, en concreto, qué tipo de actitudes lingüísticas tienen los hablantes de El Salvador hacia algunos rasgos lingüísticos de su español y del español de España, y si hay alguna correlación entre sus valoraciones y las variables género, edad, nivel de educación y nivel económico.

\section{Metodología}

Para este estudio, se realizó una encuesta en la ciudad de San Salvador a 100 participantes. El cuestionario tenía 16 afirmaciones. Cada encuesta contó con una serie de afirmaciones sobre el español salvadoreño y peninsular. En cada afirmación el participante debía seleccionar una de 5 opciones (escala de Likert):

a) $5=$ completamente de acuerdo,

b) $4=$ de acuerdo,

c) $3=$ ni de acuerdo ni en desacuerdo,

d) $2=$ un poco en desacuerdo,

e) $1=$ totalmente en desacuerdo.

Aparte de los temas relacionados con las dos variedades de español antes mencionadas, las afirmaciones también trataban algunos mitos asociados con la concordancia entre la ortografía y la pronunciación. La selección de los participantes se realizó al azar, en universidades públicas y privadas, y otros en diversos barrios, de clase media y baja, de la ciudad de San Salvador.

El procedimiento consistió primeramente en llenar, leer y firmar una hoja de consentimiento informado y otra con datos personales (edad, género, estudios y ocupación). Luego, cada participante procedió a completar el cuestionario. Todas las encuestas fueron anónimas. En el caso de aquellos participantes que no sabían leer ni escribir fue el entrevistador quien rellenó todos los materiales, y el encuestado dio oralmente su información.

Las variables que se analizaron fueron: 
- el género (50 mujeres y 50 hombres);

- la edad, agrupada en tres categorías de la siguiente manera: de 18 a 29 años (39 participantes), adultos de 30 a 49 años (44 participantes) y con más de 50 años (17 participantes);

- el nivel económico, que se dividió en dos grupos, los de un nivel bajo (65 participantes) y los de clase media (35); y finalmente,

- el nivel educativo, que se resume así: 27 participantes que tenían ningún estudio o solo estudios de primaria o educación básica (no más de 9 años de escuela), 22 más que habían terminado o estaban en bachillerato (grados 10, 11 y 12 antes de la universidad) y 51 que estaban asistiendo o habían estudiado alguna vez o terminado una carrera universitaria.

En cuanto a rasgos específicos del español del centro de España se preguntó indirectamente sobre la aspiración de $s$ y el uso del fonema fricativo interdental sordo, dado que parecen ser rasgos popularmente asociados con el acento español.

Para el análisis estadístico, se usó el programa SPSS. En cada test estadístico se consideró el nivel de probabilidad $p<.05$. Para las variables en que se contó con dos grupos (género y nivel económico), las respuestas se analizaron con el test estadístico independent samples t-test, que nos dice si las medias de dos grupos difieren significativamente. Para comparar las respuestas en las afirmaciones del cuestionario con aquellas variables que tenían 3 grupos (edad y educación), se utilizó el test estadístico one-way ANOVA. En cada caso, la lista dependiente fueron los valores que ofrecieron los participantes en cada afirmación, mientras que el factor fue el grupo. Nuestras tablas de resultados contienen información sobre el número de participantes $(\mathrm{N})$, la media de cada respuesta, la desviación estándar (que es la variación de los resultados a partir de la media, de forma que cuanto más baja sea, más uniformidad habrá en las respuestas de los participantes) y el valor $p$. Explicaremos más detalles de los resultados del programa SPSS a medida que vayamos presentando cada tabla.

\section{Resultados Y DiscusióN}

\subsection{Resultados descriptivos}

A continuación, se muestra una tabla con los resultados descriptivos de todas las afirmaciones. Posteriormente, se muestran las tablas con los resultados en que se obtuvieron diferencias significativas en el análisis estadístico. 


\begin{tabular}{|l|c|c|c|}
\hline \multicolumn{1}{|c|}{ Afirmación } & Sí & Inseguro & No \\
\hline 1. El mejor español se habla en El Salvador & $9 \%$ & $28 \%$ & $63 \%$ \\
\hline $\begin{array}{l}\text { 2. El español de El Salvador es tan válido } \\
\text { como el de otros países }\end{array}$ & $51 \%$ & $18 \%$ & $31 \%$ \\
\hline $\begin{array}{l}\text { 3. El español de El Salvador no es español } \\
\text { de verdad, es un dialecto }\end{array}$ & $25 \%$ & $24 \%$ & $51 \%$ \\
\hline $\begin{array}{l}\text { 4. El español de mi país no se lo recomendaría } \\
\text { a un extranjero que aprende español }\end{array}$ & $26 \%$ & $17 \%$ & $57 \%$ \\
\hline 5. Me siento orgulloso del acento de mi país & $72 \%$ & $12 \%$ & $16 \%$ \\
\hline $\begin{array}{l}\text { 6. Hablar con el acento de mi país me hace } \\
\text { sentir identificado con mi cultura }\end{array}$ & $74 \%$ & $14 \%$ & $12 \%$ \\
\hline 7. El mejor español se habla en España & $67 \%$ & $16 \%$ & $17 \%$ \\
\hline $\begin{array}{l}\text { 8. Uno debe pronunciar la z como se hace } \\
\text { en España }\end{array}$ & $38 \%$ & $20 \%$ & $42 \%$ \\
\hline $\begin{array}{l}\text { 9. Me gustaría que mis hijos hablaran español } \\
\text { como el de España o parecido a ese }\end{array}$ & $46 \%$ & $24 \%$ & $30 \%$ \\
\hline $\begin{array}{l}\text { 10. El presidente de El Salvador debería hablar } \\
\text { con el español de España }\end{array}$ & $10 \%$ & $21 \%$ & $69 \%$ \\
\hline $\begin{array}{l}\text { 11. Si la Real Academia Española prohibiera } \\
\text { la forma de hablar de mi país, yo haría caso } \\
\text { y aprendería lo que dijeran }\end{array}$ & $23 \%$ & $18 \%$ & $59 \%$ \\
\hline $\begin{array}{l}\text { 12. Si no aprendemos el español de España, } \\
\text { el español se va a convertir en muchos } \\
\text { dialectos }\end{array}$ & $27 \%$ & $31 \%$ & $42 \%$ \\
\hline $\begin{array}{l}\text { 13. No me gusta cuando un salvadoreño } \\
\text { regresa de otro país y viene hablando } \\
\text { con acento diferente }\end{array}$ & $60 \%$ \\
\hline $\begin{array}{l}\text { 14. Si me mudara a otro país donde se hablara } \\
\text { español, cambiaría mi acento porque } \\
\text { el de mi país no me gusta }\end{array}$ & $19 \%$ & $10 \%$ & $71 \%$ \\
\hline $\begin{array}{l}\text { 15. Uno debe diferenciar la pronunciación } \\
\text { de } b \text { y von las jocho» en vez de «son las ocho» }\end{array}$ & $72 \%$ & $14 \%$ & $12 \%$ \\
\hline $\begin{array}{l}\text { 16. Creo que está muy mal cuando alguien dice } \\
\text { «son }\end{array}$ & $29 \%$ \\
\hline
\end{tabular}

«Sí» es la suma de «completamente de acuerdo»y «de acuerdo», «no» es la suma de «un poco en desacuerdo» $\mathrm{y}$ «totalmente en desacuerdo», mientras que «inseguro» se refiere a «ni de acuerdo ni en desacuerdo»

TABLA 1: Análisis descriptivo de todas las afirmaciones $(\mathrm{N}=100)$ 


\subsubsection{Actitudes hacia el español salvadoreño}

El primer bloque de preguntas (1-6) está relacionado con la apreciación de los hablantes salvadoreños hacia su propia variedad de español. Vemos que la primera aseveración, que define el español salvadoreño como «el mejor», recibe una desaprobación del 63\%, con lo que es claro que los salvadoreños no creen que se merezca ese título. Sin embargo, las restantes cinco afirmaciones dan al español salvadoreño una buena evaluación. Por un lado, las afirmaciones que dicen que el español salvadoreño no es «español de verdad» y que no se lo recomendarían a ningún extranjero son rechazadas en un 51 y 57 por ciento, respectivamente. La apreciación positiva hacia la lengua nacional se vuelve a notar cuando los encuestados estuvieron de acuerdo en que su español es tan válido como el de otros países $(51 \%)$. Finalmente, las dos últimas afirmaciones de este bloque corroboran que los hablantes salvadoreños ven con orgullo su forma de hablar y que también se sienten identificados con ella (más de un $70 \%$ a favor).

\subsubsection{Actitudes hacia el español Peninsular}

Un $67 \%$ se mostró de acuerdo en que el «mejor español» se habla en España, estimación que concuerda con la apreciación de que el español salvadoreño no lo es. Los hablantes también estuvieron de acuerdo, aunque en menor medida, en que les gustaría que sus hijos hablaran el español peninsular o uno parecido. En ese sentido, tal parece que el español de España tiene una buena percepción. En cuanto a la afirmación 8 , la opinión se ve relativamente dividida en cuanto a pronunciar la $z$ como en el norte de España. Sin embargo, los participantes no aprobaron que el presidente hablara con un español peninsular. Tampoco estuvieron de acuerdo en seguir las normas de la Real Academia Española. Los participantes tampoco piensan, en su mayoría, que el español se vaya a convertir en varios dialectos si no se aprende el de España. Estos resultados coinciden con nuestra propuesta de que el prestigio del español de España no anima a los salvadoreños a imitarlo, como se esperaría si ese prestigio fuera real.

\subsubsection{La adopción de otros acentos}

Se quería saber si los participantes ven con buenos ojos cuando un salvadoreño adopta un acento extranjero, sobre todo al regresar del extranjero. El estar de acuerdo con ello sería un signo de que cualquier otra variedad es mejor que la salvadoreña. En este respecto, como se 
observa en la aseveración 13, el $60 \%$ estuvo de acuerdo en que no le gustaría. De la misma forma, y de manera más contundente, el $71 \%$ de los encuestados se mostraron en desacuerdo en que cambiarían su acento porque el de su país no les gusta.

\subsubsection{La ortografía y la pronunciación}

Estas afirmaciones intentan indagar en las actitudes sobre la relación entre la escritura y la pronunciación. En la afirmación 8 hemos visto ya que los participantes se mostraron ambivalentes en cuanto a pronunciar la $z$ como en el norte de España, lo que podría ser señal de estigmatización hacia ese sonido, bastante ajeno al español salvadoreño. En las otras dos afirmaciones sobre este tema los participantes fueron más directos en apoyar la idea de "pronunciar las letras tal y como se escriben». Ese fue el caso de la diferencia entre $b$ y $v(74 \%)$ y la aspiración de $s(72 \%)$. La aspiración, muy generalizada en el habla salvadoreña, parece estar muy estigmatizada según este resultado. Hecho que se puede apreciar en el habla cuidada y en los medios de comunicación salvadoreños, donde se intenta evitar (aunque con poco éxito).

\subsection{Resultados estadísticos}

A continuación se muestran los resultados de aquellas afirmaciones que mostraron diferencias significativas. Estos resultados se analizan de acuerdo con cada variable.

\subsubsection{La variable género}

En esta variable no hubo resultados significativos entre los diferentes grupos en ninguna de las afirmaciones. Por lo tanto, esta variable no constituye un factor en cuanto a las actitudes de los participantes, ya fueran positivas o negativas. Tal parece que las opiniones de los salvadoreños, al menos en cuanto al factor género, son bastante homogéneas.

\subsubsection{La variable edad}

Entre los diferentes grupos de esta variable, solo se encontraron diferencias significativas en una sola afirmación, la número dos, en la que se señala que el español de El Salvador es tan válido como el de otros países (ANOVA $F(2,97)=6.066, p<.005)$. Vemos que hay una diferencia 
significativa entre el grupo de jóvenes y el de adultos, siendo estos últimos quienes ven el español salvadoreño tan válido como otras variedades. Pensamos que esto podría ser señal de un aumento de inseguridad lingüística entre los jóvenes, debido a que el acceso a la educación se ha ido incrementando recientemente.

\begin{tabular}{|c|c|c|c|c|c|c|c|c|c|}
\hline \multicolumn{7}{|c|}{ One-way ANOVA } & \multicolumn{3}{|c|}{ Scheffe post hoc } \\
\hline & Grupo & $\mathrm{N}$ & Media & $\begin{array}{c}\text { Desviación } \\
\text { estándar }\end{array}$ & $\mathrm{F}$ & $p$ & $\begin{array}{c}\text { Diferencia } \\
\text { medias }\end{array}$ & $\begin{array}{c}\text { Error } \\
\text { estándar }\end{array}$ & $p$ \\
\hline (J) & 18 a 29 & 39 & 2.79 & 1.281 & \multirow[t]{3}{*}{6.066} & \multirow[t]{3}{*}{.003} & $\mathrm{~J}-\mathrm{A}$ & .282 & .117 \\
\hline (A) & 30 a 49 & 44 & 3.39 & 1.401 & & & $\mathrm{~J}-\mathrm{M}$ & .373 & .004 \\
\hline (M) & 50 o más & 17 & 4.06 & .899 & & & $A-M$ & .366 & .191 \\
\hline
\end{tabular}

TABLA 2: Resultados de la ANOVA con Scheffe post hoc para la afirmación «El español de El Salvador es tan válido como el de otros países», en cuanto a la variable EDAD

\subsubsection{La variable nivel económico}

Dentro de esta variable, se encontraron diferencias significativas entre los diferentes grupos en dos de las afirmaciones que se hicieron en el cuestionario. La primera afirmación que proporcionó resultados significativos $(p<.05)$ entre los participantes de nivel económico bajo $(\mathrm{N}=65, \mathrm{M}=2.32, \mathrm{SD}=1.077)$ y los de nivel económico medio $(\mathrm{N}=35$, $\mathrm{M}=1.77, \mathrm{SD}=.973)$ fue la que decía que el mejor español se habla en El Salvador.

\begin{tabular}{|c|c|c|c|c|c|c|}
\hline $\begin{array}{c}\text { Nivel } \\
\text { económico }\end{array}$ & $\mathrm{N}$ & Media & $\begin{array}{c}\text { Desviación } \\
\text { estándar }\end{array}$ & $\begin{array}{c}\text { Grado } \\
\text { de libertad }\end{array}$ & Valor T & $p$ \\
\hline Bajo & 65 & 2.32 & 1.077 & 98 & 2.525 & .013 \\
\cline { 1 - 4 } Medio & 35 & 1.77 & .973 & & \\
\hline
\end{tabular}

TABLA 3: Resultados del t-test para la afirmación «El mejor español se habla en El Salvador», con la variable NIVEL ECONÓMICO

Es interesante ver que son los hablantes de nivel económico bajo quienes muestran más apoyo a esta idea (si se comparan con los de nivel económico superior), aunque en general la mayoría se opuso. 
También se encontraron resultados significativos $(p<.05)$ entre los participantes de nivel económico bajo $(\mathrm{N}=65, \mathrm{M}=4.18, \mathrm{SD}=1.059)$ y los de nivel económico medio $(\mathrm{N}=35, \mathrm{M}=3.69, \mathrm{SD}=1.451)$ en la afirmación que sostenía que uno debe diferenciar la pronunciación de $b$ y $v$. En este caso, son los hablantes del nivel económico bajo quienes más favorecen esta aseveración.

\begin{tabular}{|c|c|c|c|c|c|c|}
\hline $\begin{array}{c}\text { Nivel } \\
\text { económico }\end{array}$ & $\mathrm{N}$ & Media & $\begin{array}{c}\text { Desviación } \\
\text { estándar }\end{array}$ & $\begin{array}{c}\text { Grado } \\
\text { de libertad }\end{array}$ & Valor T & $p$ \\
\hline Bajo & 65 & 4.18 & 1.059 & 98 & 1.968 & .052 \\
\cline { 1 - 3 } Medio & 35 & 3.69 & 1.451 & & & \\
\hline
\end{tabular}

TABLA 4: Resultados del $t$-test para la afirmación «Uno debe diferenciar la pronunciación de $b$ y $v »$, con la variable NIVEL ECONÓMICO

\subsubsection{La variable nivel educativo}

En esta variable hubo cinco afirmaciones que mostraron resultados de interés, lo que demuestra la importancia de la educación en las actitudes que tratamos. En cuanto a la afirmación que decía que el mejor español del mundo se habla en El Salvador, las pruebas estadísticas mostraron que había una diferencia significativa (ANOVA $F(2,97)=7.772$, $p<.005$ ) entre los tres grupos (con estudios de primaria o menos, con bachillerato y con estudios universitarios).

\begin{tabular}{|c|c|c|c|c|c|c|c|c|c|}
\hline \multicolumn{7}{|c|}{ One-way ANOVA } & \multicolumn{3}{|c|}{ Scheffe post hoc } \\
\hline & Grupo & $\mathrm{N}$ & Media & $\begin{array}{c}\text { Desviación } \\
\text { estándar }\end{array}$ & $\mathrm{F}$ & $p$ & $\begin{array}{c}\text { Diferencia } \\
\text { medias }\end{array}$ & $\begin{array}{c}\text { Error } \\
\text { estándar }\end{array}$ & $p$ \\
\hline$(\mathrm{P})$ & Primaria & 27 & 2.78 & 1.155 & 7.772 & .001 & $P-B$ & .288 & .020 \\
\hline (B) & Bachillerato & 22 & 1.95 & .999 & & & $\mathrm{P}-\mathrm{U}$ & .239 & .001 \\
\hline (U) & Universidad & 51 & 1.86 & .917 & & & $\mathrm{~B}-\mathrm{U}$ & .256 & .938 \\
\hline
\end{tabular}

TABLA 5: Resultados de la ANOVA con Scheffe post hoc para la afirmación «El mejor español se habla en El Salvador», en cuanto a la variable EDUCACIÓN

Como vimos en los resultados descriptivos, la mayoría de participantes no estuvo de acuerdo con esta aseveración. Las pruebas estadísticas 
revelan que hay más diferencias entre el grupo con escolaridad básica y el de estudios universitarios, siendo estos últimos quienes menos favorecen esta idea de que el mejor español sea el salvadoreño.

Igualmente, en la afirmación que sostenía que el español de El Salvador es tan válido como el de otros países, las pruebas estadísticas mostraron que había una diferencia significativa (ANOVA $F(2,97)=5.335$, $p<.01)$ entre los tres grupos.

\begin{tabular}{|c|c|c|c|c|c|c|c|c|c|}
\hline \multicolumn{7}{|c|}{ One-way ANOVA } & \multicolumn{3}{|c|}{ Scheffe post hoc } \\
\hline & Grupo & $\mathrm{N}$ & Media & $\begin{array}{l}\text { Desviación } \\
\text { estándar }\end{array}$ & $\mathrm{F}$ & $p$ & $\begin{array}{c}\text { Diferencia } \\
\text { medias }\end{array}$ & $\begin{array}{c}\text { Error } \\
\text { estándar }\end{array}$ & $p$ \\
\hline (P) & Primaria & 27 & 3.93 & .997 & \multirow[t]{3}{*}{5.335} & \multirow[t]{3}{*}{.006} & $\mathrm{P}-\mathrm{B}$ & .371 & .218 \\
\hline (B) & Bachillerato & 22 & 3.27 & 1.518 & & & $\mathrm{P}-\mathrm{U}$ & .307 & .006 \\
\hline (U) & Universidad & 51 & 2.92 & 1.324 & & & $B-U$ & .330 & .569 \\
\hline
\end{tabular}

TABLA 6: Resultados de la ANOVA con Scheffe post hoc para la afirmación «El español de El Salvador es tan válido como el de otros países», en cuanto a la variable EDUCACIÓN

Vemos que la diferencia está más enfatizada entre los que tienen pocos estudios y los universitarios, siendo estos últimos quienes menos apoyan la validez del español salvadoreño. Una vez más, es evidente que con la educación, los salvadoreños parecen apreciar menos su variedad de español.

Otra afirmación en que las pruebas estadísticas mostraron una diferencia significativa (ANOVA $F(2,97)=4.965, p<.01$ ) entre los tres grupos fue la que expresaba que el español de El Salvador no se le recomendaría a un extranjero que aprende español.

\begin{tabular}{|c|c|c|c|c|c|c|c|c|c|}
\hline \multicolumn{7}{|c|}{ One-way ANOVA } & \multicolumn{3}{|c|}{ Scheffe post hoc } \\
\hline & Grupo & $\mathrm{N}$ & Media & $\begin{array}{l}\text { Desviación } \\
\text { estándar }\end{array}$ & $\mathrm{F}$ & $p$ & $\begin{array}{c}\text { Diferencia } \\
\text { medias }\end{array}$ & $\begin{array}{c}\text { Error } \\
\text { estándar }\end{array}$ & $p$ \\
\hline$(\mathrm{P})$ & Primaria & 27 & 3.15 & 1.406 & 4.965 & .009 & $\mathrm{P}-\mathrm{B}$ & .392 & .012 \\
\hline (B) & Bachillerato & 22 & 1.95 & 1.214 & & & $\mathrm{P}-\mathrm{U}$ & .325 & .072 \\
\hline (U) & Universidad & 51 & 2.39 & 1.401 & & & $\mathrm{~B}-\mathrm{U}$ & .348 & .456 \\
\hline
\end{tabular}

TABLA 7: Resultados de la ANOVA con Scheffe post hoc para la afirmación «El español de mi país no se lo recomendaría a un extranjero que aprende español», en cuanto a la variable EDUCACIÓN 
El grupo que más apoyó esta aseveración fue el de menor nivel de estudios, mientras que los grupos con mayor formación no parecen favorecerla.

Las pruebas estadísticas también mostraron que había una diferencia significativa (ANOVA $F(2,97)=4.228, p<.05$ ) entre los tres grupos de esta variable con respecto a la afirmación «Me siento orgulloso del acento de mi país».

\begin{tabular}{|c|c|c|c|c|c|c|c|c|c|}
\hline \multicolumn{7}{|c|}{ One-way ANOVA } & \multicolumn{3}{|c|}{ Scheffe post hoc } \\
\hline & Grupo & $\mathrm{N}$ & Media & $\begin{array}{l}\text { Desviación } \\
\text { estándar }\end{array}$ & $\mathrm{F}$ & $p$ & $\begin{array}{c}\text { Diferencia } \\
\text { medias }\end{array}$ & $\begin{array}{c}\text { Error } \\
\text { estándar }\end{array}$ & $p$ \\
\hline (P) & Primaria & 27 & 4.37 & .967 & \multirow[t]{3}{*}{4.228} & \multirow[t]{3}{*}{.017} & $\mathrm{P}-\mathrm{B}$ & .338 & .712 \\
\hline (B) & Bachillerato & 22 & 4.09 & 1.109 & & & $\mathrm{P}-\mathrm{U}$ & .280 & .024 \\
\hline (U) & Universidad & 51 & 3.59 & 1.299 & & & $\mathrm{~B}-\mathrm{U}$ & .300 & .251 \\
\hline
\end{tabular}

TABLA 8: Resultados de la ANOVA con Scheffe post hoc para la afirmación «Me siento orgulloso del acento de mi país», en cuanto a la variable EDUCACIÓN

Podemos observar que son, una vez más, los participantes con menos escolaridad quienes mayor aprecio muestran hacia el español nacional. Si bien en los resultados descriptivos sobre esta aseveración se muestra que casi todos los salvadoreños parecen estar orgullosos de su acento, tal parece que la educación les hace ver que hablar como salvadoreño es «malo».

Finalmente, las pruebas estadísticas encontraron que también había una diferencia significativa (ANOVA $F(2,97)=5.254, p<.01$ ) entre los tres grupos sobre la afirmación que indicaba que a los participantes les gustaría que sus hijos hablaran español como el de España o parecido a ese.

\begin{tabular}{|c|c|c|c|c|c|c|c|c|c|}
\hline \multicolumn{7}{|c|}{ One-way ANOVA } & \multicolumn{3}{|c|}{ Scheffe post hoc } \\
\hline & Grupo & $\mathrm{N}$ & Media & $\begin{array}{c}\text { Desviación } \\
\text { estándar }\end{array}$ & $\mathrm{F}$ & $p$ & $\begin{array}{c}\text { Diferencia } \\
\text { medias }\end{array}$ & $\begin{array}{c}\text { Error } \\
\text { estándar }\end{array}$ & $p$ \\
\hline$(\mathrm{P})$ & Primaria & 27 & 3.59 & 1.279 & \multirow[t]{3}{*}{5.254} & \multirow[t]{3}{*}{.007} & $P-B$ & .368 & .971 \\
\hline (B) & Bachillerato & 22 & 3.68 & 1.249 & & & $\mathrm{P}-\mathrm{U}$ & .305 & .039 \\
\hline$(\mathrm{U})$ & Universidad & 51 & 2.80 & 1.296 & & & $\mathrm{~B}-\mathrm{U}$ & .327 & .031 \\
\hline
\end{tabular}

TABLA 9: Resultados de la ANOVA con Scheffe post hoc para la afirmación «Me gustaría que mis hijos hablaran español como el de España o parecido a ese», en cuanto a la variable EDUCACIÓN 
Los resultados de la Tabla 9 muestran que los participantes que tienen menos educación son los que quisieran que sus hijos adoptaran un acento español, a pesar de que son ellos mismos los que manifiestan mayor orgullo por el acento salvadoreño. Los hablantes con más educación, por el contrario, apoyan menos la idea de adoptar un español castizo, aunque en las otras aseveraciones favorecían el español de España. No hay duda, según nuestros resultados, de que los hablantes salvadoreños padecen de inseguridad lingüística, y que esta inseguridad se ve aumentada cuando entra en juego el factor educación.

\section{Conclusiones}

Vemos en los resultados descriptivos que de seis afirmaciones que se hacían sobre el español salvadoreño, solo una, la que considera el español salvadoreño como el mejor del mundo, recibió una valoración negativa. Por otro lado, que de las seis afirmaciones que trataban sobre el español peninsular, cuatro fueron vistas negativamente y dos positivamente, siendo la que postulaba al español peninsular como el mejor la que mayor aceptación consiguió. Está claro que la idea de que el mejor español se habla en España sigue teniendo pujanza entre los salvadoreños. Otras dos afirmaciones relacionadas con «cambiar el español salvadoreño» por otro fueron también evaluadas negativamente (13 y 14). Por otro lado, la distinción fonética entre $b$ y $v$ fue evaluada positivamente, mientras que la aspiración de /s/, tan común en El Salvador, fue rechazada por la mayoría. Esto implica que la relación entre la ortografía y la pronunciación sigue siendo vista como relevante. Es interesante, además, que la distinción entre $s$ y $z$ no es bien vista si es entre salvadoreños, a pesar de que está relacionada con la idea de concordancia entre la escritura y la pronunciación.

En cuanto a las variables sociales, la que no presentó ninguna diferencia significativa fue el género, mientras que el nivel de educación fue lo que más influyó en las respuestas de los participantes. Este resultado es importante porque confirma que la educación puede ser la causa de la inseguridad lingüística entre los salvadoreños. Cuanto mayor es el nivel de estudios, más incertidumbre hay con respecto a la forma de hablar local. Es posible, sin embargo, que no podamos hablar en este caso de una «esquizofrenia actitudinal», como trata Bañón 1993, p. 260, ya que los resultados descriptivos, a nivel general, también muestran señales positivas de este grupo hacia el español salvadoreño.

En cuanto a la edad, podemos concluir que los adultos (no los mayores) son los que piensan que el español salvadoreño es tan válido como el de otras regiones y los que están también de acuerdo en que el mejor español se habla en El Salvador. Asimismo, este grupo también apoya 
en mayor medida que se haga diferencia entre la pronunciación de $b$ y $v$. El grupo con más educación, por su lado, estuvo menos de acuerdo en que el mejor español sea el salvadoreño, así como que este sea tan válido como el de otras regiones y también fue este grupo el que menos orgulloso está de su acento salvadoreño. Curiosamente, este grupo estuvo menos de acuerdo en que sus hijos aprendieran el español de España, lo que fortalece la idea que mencionamos antes (la educación como fuente de inseguridad lingüística).

Como hacemos notar en nuestro título, este trabajo no es más que un esbozo de las actitudes lingüísticas en El Salvador. Esperamos que estos resultados animen a otros lingüistas a estudiar el olvidado español salvadoreño.

\section{REFERENCIAS BIBLIOGRÁFICAS}

Asociación de ACAdemias de la Lengua Española (2010): Diccionario de americanismos, Madrid, Alfaguara.

BAÑóN, A. (1993): «Adolescencia, variación lingüística, competencia metacomunicativa y enseñanza de la lengua», Tonos digital: Revista electrónica de estudios filológicos 9, pp. 253-285.

Bello, A. (1970): Antología de Andrés Bello: advertencias sobre el uso de la lengua castellana, dirigidas a los padres de familia, profesores de los colegios y maestros de escuela, Caracas, Ministerio de Educación.

- y Cuervo, R. (1948): Gramática de la lengua castellana. Nueva edición hecha sobre la última del autor con extensas notas y copiosos indices alfabéticos, Buenos Aires, Ediciones Anaconda.

Ferguson, C. (1959): «Diglossia», Word 15, pp. 325-403.

GUITART, J. (1996): «Spanish in contact with itself and the phonological characterization of conservative and radical styles», en Roca, A. y Jensen, J. (eds.), Spanish in contact, issues in bilingualism, Sommerville, Cascadilla Press, pp. 151-157.

Haugen, E. (1962): «Schizoglossia and the linguistic norm», en Dil, A. S. (ed.), The ecology of language. Essays by Enair Haugen, Stanford, Stanford University Press, pp. 148-158.

HERNÁNDEZ, J. (2002): «Accommodation in a dialect contact situation», Revista de Filología y Linguistica de la Universidad de Costa Rica 28/2, pp. 93-110.

— y JIMÉNEZ, J. (2003): «Broadcasting standardization: An analysis of the linguistic normalization process in Murcian Spanish», Journal of Sociolinguistics 7/3, pp. 321-347.

HERNÁNDEZ-CAMPOY, J. (2003): «Complementary approaches to the diffusion of standard features in a local community», en Britain, D. y Cheshire, J. (eds.), Social dialectology: In honor of Peter Trudgill, Ámsterdam, Benjamins.

LARA, L. (2001): «El español como lengua americana», Revista de Occidente 245, pp. 31-36. 
LIPSKI, J. (1986): «Central American Spanish in the United States: some remarks on the Salvadoran community», Aztlán 17, pp. 91-123.

- (1994): Latin American Spanish, Londres, Addison Wesley Publishing Company (trad. esp.: El español de América, Madrid, Cátedra, 2006).

Lope Blanch, J. (1988): «La labiodental sonora en el español de México», Nueva Revista de Filología Hispánica 36/1, pp. 153-170.

LÓPEz, H. (2000): «Rasgos generales», Manual de dialectología hispánica, el español en América, Madrid, Editorial Ariel.

- (2001): «Actitudes lingüísticas hacia el bable en la ciudad de Oviedo», Lingüistica Española Actual 23/2, pp. 145-158.

QUiNTANILLA, J. (2005): «Reconsideraciones sobre la delimitación y el uso del voseo americano», Cuadernos Cervantes de la Lengua Española 11, 59-60, pp. 66-74.

- (2009): «Actitudes de los hablantes de San Salvador hacia el tuteo y el voseo», Hispania 92/2, pp. 361-373.

RIvas, P. (1978): La lengua salvadoreña, San Salvador, Ministerio de Educacion, Direccion de Publicaciones.

Stevens, J. (2000): «On the labiodental pronunciation of Spanish /b/ among teachers of Spanish as a second language», Hispania 83/1, pp. 139-149.

Velleman, B. (1997): «Domingo F. Sarmiento y la función social de la lengua», Historiographia Linguistica 24/1-2, pp. 159-174. 
